\title{
Effective non-retarded method as a tool for the design of tunable nanoparticle composite absorbers
}

\author{
Guillermo Ortiz, ${ }^{1}$ Marina Inchaussandague, ${ }^{2}$ Diana Skigin, ${ }^{2}$ \\ Ricardo Depine, ${ }^{2}$ and Luis Mochán ${ }^{3}$ \\ ${ }^{1}$ Departamento de Física, Facultad de Ciencias Exactas, Naturales y Agrimensura, \\ Universidad Nacional del Nordeste, \\ Avenida Libertad 5470, W3404AAS Corrientes, Argentina \\ ${ }^{2}$ Grupo de Electromagnetismo Aplicado, Departamento de Física, FCEN, UBA \\ and IFIBA, CONICET, Pabellón 1, Ciudad Universitaria, 1428 Buenos Aires, \\ Argentina \\ ${ }^{3}$ Instituto de Ciencias Físicas, Universidad Nacional Autónoma de México, \\ Apdo. Postal 48-3, 62251 Cuernavaca, Morelos, México \\ E-mail: gortiz@unne.edu.ar
}

\begin{abstract}
We investigate the capabilities of the effective non-retarded method (ENR) to explore and design nanoparticles composites with specific optical properties. We consider a composite material comprising periodically distributed metallic spheres in a dielectric host matrix. The effective macroscopic dielectric function of the composite medium is obtained by means of the ENR and is used to calculate the electromagnetic response of a slab made of such an inhomogeneous material. This response is compared with that obtained using the Korringa-Kohn-Rostoker wave calculation method (KKR). We analyze the optical properties for different filling fractions, especially in the vicinity of the resonance frequencies of the macroscopic dielectric function. We show that appropriately choosing the parameters of the composite it is possible to achieve a tunable absorber film. The ENR results to be a versatile tool for the design of nanoparticle composite materials with specific properties.
\end{abstract}

PACS numbers: 78.67.Bf, 77.22.Ch, 78.20.Ci, 78.20.Bh

\section{Introduction}

The optical properties of inhomogeneous material systems have been extensively studied for the last decades. The formulations developed, such as the theories of spectral representations [1, 2, 3], spatial fluctuations [4, 5], renormalized polarizabilities [6, 7] and diagrammatic series [8, 9], intended to explain the physical phenomena involved in the electromagnetic macroscopic response of these systems using analytical and/or semianalytical expressions. However, since the emergence and development of computational tools, implementations such as the Discrete Dipole Approximation [10, 11], and the Finite-difference time-domain (FDTD) method [12, 13], have gained widespread interest. The contribution of these developments has been primarily focused on the 
corroboration of the transmission/reflection spectra obtained experimentally in complex systems. Modern technology possibilities of miniaturization and self-assembling have accompanied these developments [14, 15].

If the typical wavelengths are much larger than the size of the inhomogeneities, it is possible to make a quasi-static treatment (also called long wavelength approximation), in which many of the current methods for determining the effective electric macroscopic permittivity of the system are based. An enriching review of traditional approaches to solve the problem by means of effective medium models can be found in [16, 17, 18. If the wavelength is comparable to the size of the inhomogeneities, it is still possible to use effective medium models to obtain the macroscopic dielectric function of metal-dielectric composites [19, 20], although in these cases the dispersion of the dielectric response is both spatial and temporal. We are interested in investigating inhomogeneous systems formed by two ordered phases, one metallic and the other dielectric. The interest in metal-dielectric composites lies in the possibility of designing their optical properties by adapting the parameters of the materials. More specifically, if the phases of the composite are chosen with appropriate geometry and sizes, it is possible to tune the resonance of the dielectric macroscopic function within the visible range [21, 22, 23].

Effective medium theories describe the macroscopic dielectric function of complex composite nanostructures in terms of the dielectric functions of their components and of a limited number of geometrical parameters. In the case of diluted systems with spherical inclusions, the filling fraction is sufficient information to obtain the macroscopic dielectric function, as established by the traditional effective medium theories [24, 25]. However, most of the interesting effects are more strongly manifested for inclusions with complex geometries and close to the percolation threshold of the conductive phase, i.e. in systems with high filling fractions. As a result, it raises the question of how these formulations should be modified to account for the effects of more complex and concentrated structures. It is well known that when the metallic particles which are close enough, multipolar interactions among them give rise to a dielectric macroscopic response that exhibits several resonances, regardless of the inclusions'size [26, 27]. However, following the results by Waterman et. al. [28], many authors have neglected the multipolar order contribution [29], even for filling fraction near the close packing condition [30]. This assumption has also been proposed for the development of composite system with novel optical properties [31.

Among the theories developed to study non-diluted [32, 33] and ordered [27, 34, 21] systems, the effective non-retarded method (ENR) has been recently proposed. This formalism makes it possible to obtain the complex and frequency dependent macroscopic dielectric function for arbitrarily shaped inclusions periodically ordered in 2D or 3D arrays. It can also deal with interpenetrating inclusions and dissipative and dispersive materials [22]. Although the ENR allows dealing with inclusions of arbitrary geometry, it is particularly suited for particles with planar faces such as cubes or parallelepipeds. This is because its numerical implementation requires a discrete representation of the scatterers, for which a cubic grid is used to discretize the unit cell. Given the 
demonstrated versatility of this method for predicting the optical properties of complex materials [23, it is important to study its behavior when applied to the case of composites with spherical inclusions, as those that have been recently manufactured [35, 36, 37]. The case of spherical inclusions requires a detailed analysis of the numerical procedure employed to determine the dielectric macroscopic function. Moreover, it is essential to validate the results by comparison with rigorous methods which do not include approximations in the representation of the shape of the inclusions. Among these methods, the KKR makes it possible to investigate the electromagnetic response of composites formed by periodic arrays of spheres and was shown to be numerically efficient. In the KKR, the electromagnetic interactions between the scatterers are calculated by means of the layer-multiple-scattering method for spherical particles [38, 39, 40, 41]. Recently, simulated reflectance spectra calculated by means the vector KKR at high order band frequencies, have shown a clear correlation between theoretical and experimental results [42. It is our aim to study the applicability of the ENR to the case of composites formed by spherical metallic inclusions embedded in a dielectric medium. To do so, we investigate the electromagnetic response of a composite slab and compare the results obtained using the ENR and the KKR. In Section 2, the ENR is used to obtain the macroscopic dielectric response of a medium comprising a simple cubic lattice of spheres. In Section 3, the KKR is used to obtain the electromagnetic response of a composite material slab and it is compared with that given by the ENR. In Section 4 we explore the potential of the ENR as a design tool for tunable absorbers from diluted to overlapping particles. Finally, concluding remarks are provided in Section 5 .

\section{The macroscopic dielectric function}

Let us consider a composite made up of arbitrarily shaped inclusions arranged in a periodic 3D array. Provided that the length scale of the inclusions in the material is small compared with the electromagnetic wavelength, this mixture can be treated as a homogenized composite material. The ENR permits calculating the frequencydependent macroscopic dielectric function of such composites. It is based on Haydock's recursive scheme [43] from which a tridiagonal representation of a characteristic function [22, 23] allows to obtain the macroscopic dielectric function $\left(\epsilon^{M}\right)$ of the composite. It depends on the shape of the inclusions, on the dielectric function of the components and on the filling fraction $(f)$ of the array (ratio of the volume occupied by particles into the unit cell to the total volume of the unit cell). Since the coefficients that appear in the tridiagonal representation depend on the shape of the particles and on $f$, but not on the dielectric constant of the inclusions, once they are obtained for a particular geometry and concentration of the scatterers they can be used for inclusions of different materials, saving computation time.

In what follows, we apply the ENR to investigate materials with spherical inclusions arranged in a simple cubic lattice of period $L$. Therefore, a unit cell of the composite material is a cube of side $L$ with the inclusion at its center (Fig. 1). We consider an 


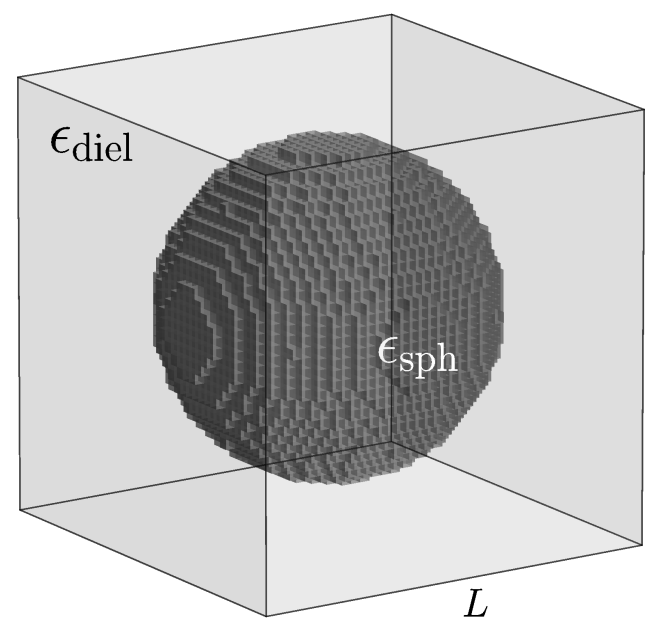

Figure 1. Unit cell of a simple cubic lattice of spherical particles of radius $r=0.38 \mathrm{~L}$ ( $L$ being the side length of the unit cell) and dielectric function $\epsilon_{\mathrm{sph}}$ embedded in a matrix with dielectric constant $\epsilon_{\text {diel }}$. The sphere is discretized by small cubes of side $L /(2 M+1)$ (in this scheme, $M=20$ ).

array of metallic spheres embedded in a dielectric host material of permittivity $\epsilon_{\text {diel }}$. To model the permittivity of the metal $\epsilon_{\mathrm{sph}}$ as a function of frequency $\omega$, we use the Drude formula

$$
\epsilon_{\mathrm{sph}}(\omega)=1-\frac{\omega_{p}^{2}}{\omega^{2}+i \omega \Gamma},
$$

where $\omega_{p}$ is the bulk plasma frequency, $\Gamma$ stands for the relaxation time and $i$ is the imaginary unit.

For the numerical implementation, the unit cell is divided into $(2 M+1)^{3}$ cubes of side $L /(2 M+1)$, by taking $2 M+1$ equidistant points on each of its sides, and the discretized inclusion is defined by the set of cubes contained within the volume of the actual sphere. The discretization process is particularly suitable for particles with planar facets, since they can be exactly represented by stacking cubes. For curved inclusions, the discretized particle exhibits a stepped surface (as shown in Fig. 1 for $M$ $=20$ ) that smooths by increasing $M$, i.e, by increasing the number of cubes and thus by decreasing their individual volume. The choice of $M$ is not only important to properly represent the geometry of the particle but also to obtain an acceptable resolution in the Fourier transform calculations involved in the ENR [22]. The value of $M$ that produces a valid description of the electromagnetic problem must be found for each combination of parameters. In Fig. 2 we show the real and imaginary parts of the macroscopic dielectric function as a function of the photon energy $\hbar \omega$ within the optical range for $M=20,60$ and 120. We consider two different composites: one made of silver particles $(\hbar \Gamma=0.03$ $\mathrm{eV}$ and $\left.\hbar \omega_{p}=8.5 \mathrm{eV}\right)$ embedded in a titanium dioxide matrix $\left(\epsilon_{\text {diel }}=7.84\right)$ (a), and the other made of gold particles $\left(\hbar \Gamma=0.1 \mathrm{eV}\right.$ and $\left.\hbar \omega_{p}=7.0 \mathrm{eV}\right)$ immersed in tellurium dioxide $\left(\epsilon_{\text {diel }}=5.2\right)(\mathrm{b})$. The values of $\hbar \omega_{p}$ and $\hbar \Gamma$ considered in the examples were obtained by fitting the real and imaginary parts of the dielectric function given by (1) 
to the experimental data of Ref. [44] in the NIR-VIS range. Two different filling fractions are considered in both cases: $f=0.045$ (left panels) and $f=0.23$ (right panels). For a simple cubic lattice, $f=(4 / 3) \pi(r / L)^{3}$, and therefore, $r / L=0.22$ for $f=0.045$ and $r / L=0.38$ for $f=0.23$. In the figure, we also include the curves obtained by means of the Maxwell-Garnett approach (MG). As shown in Fig. 2(a), the curves exhibit a sort of ripple that smooths as $M$ is increased. This fluctuation is more significant for the composite of silver spheres than for that of gold spheres (Fig. 2(b)), suggesting that the choice of a proper value of $M$ is more critical when the contrast between the permittivities of the constituent materials is higher and also for smaller values of $\Gamma$. Since the memory requirements for computing the coefficients of the tridiagonal representation of the characteristic function of the composite increase with $(2 M+1)^{3}$, in what follows we have chosen $M=120$, which is the maximum allowable value of $M$ for serial calculations [45]. This choice seems to be appropriate from the point of view of the first element of the tridiagonal matrix: since this coefficient is the average of the characteristic function within the unit cell [23], its value should tend to $f$ as $M$ increases. Taking $M=120$ the relative error of this coefficient is $\approx 0.04 \%$ for $f=0.045$. Since for a fixed value of $M$ unit cells with larger $f$ are better represented than those with smaller $f$, a relative error smaller than $0.04 \%$ is guaranteed for $f \geq 0.045$.

From Mie theory, an isolated sphere is expected to exhibit multipolar resonances for $\epsilon_{\mathrm{sph}}=-\epsilon_{\text {diel }}(l+1) / l$, where $l$ is a positive integer [46]. For $\epsilon_{\mathrm{sph}}$ given by Eq. (1), the resonant frequencies are

$$
\omega \approx \sqrt{\omega_{p}^{2} /\left(1+\epsilon_{\mathrm{diel}}(l+1) / l\right)}
$$

For the composite system, these resonances can be visualized as peaks in the imaginary part of the macroscopic dielectric function. For $f=0.045$, the curve of $\operatorname{Im}\left\{\epsilon^{M}\right\}$ exhibits a peak close to $\hbar \omega=2 \mathrm{eV}$, which for $f=0.23$ appears at $\approx 1.71 \mathrm{eV}$. These peaks are associated to the dipole resonance $(l=1)$, for which equation (2) predicts a resonant value at $\hbar \omega \approx 2.08 \mathrm{eV}$ for both composites considered in Fig. 2. As expected, this value is in very good agreement with the spectral position of the peak for the diluted system $(f=0.045)$. However, for $f=0.23$ the multiple scattering between the spheres has a significant impact on the optical response of the system and consequently, the resonant frequency is shifted with respect to the location predicted by Eq. (2) for an isolated sphere. In this case, $\operatorname{Im}\left\{\epsilon^{M}\right\}$ exhibits also another peak at $\approx 2.5 \mathrm{eV}$ which is associated to an octupole resonance $(l=3$ in Eq. (2) $)$ [27. Notice that only resonances with odd $l$ can be excited due to the system symetry. This peak does not appear in the MG curves since this approach neglects higher order interactions between the scatterers, and therefore, it is only suitable for treating diluted systems. The overall matching of the ENR and MG curves is very good. However, the dipole resonance peak obtained by the ENR is lower and slightly wider than that predicted by the MG and this can be explained by taking into account the discretized nature of the spherical particle within the ENR. In all cases, $\operatorname{Re}\left\{\epsilon^{M}\right\}$ and $\operatorname{Im}\left\{\epsilon^{M}\right\}$ satisfy the Kramers-Kroning relationship, as expected. 

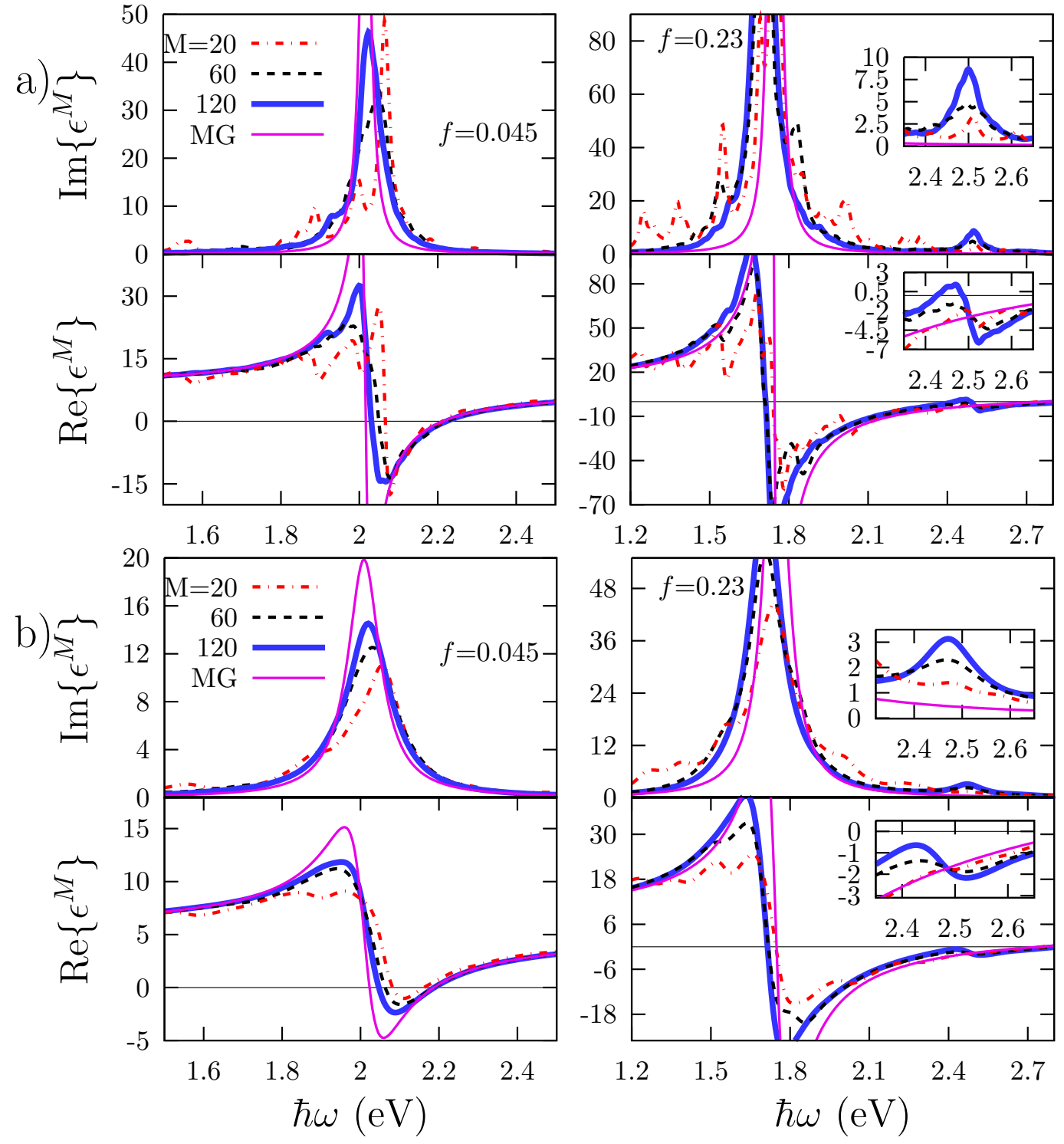

Figure 2. Macroscopic dielectric function of a composite of metallic spheres embedded in a dielectric matrix. a) Silver spheres, $\epsilon_{\text {diel }}=7.84$; b) gold spheres, $\epsilon_{\text {diel }}=5.2$. Top (bottom) panel shows the imaginary (real) part. Metallic particles are arranged in a simple cubic lattice with $f=0.045$ for the left side and $f=0.23$ for the right one. Dashed-dotted lines, dashed lines, and thick solid lines correspond to $M=20,60$ and 120, respectively. For $f=0.23$, a detail of each curve in the vicinity of $\hbar \omega \approx 2.5 \mathrm{eV}$ is displayed as an inset. Results given by the Maxwell-Garnett model are shown in thin solid lines. 


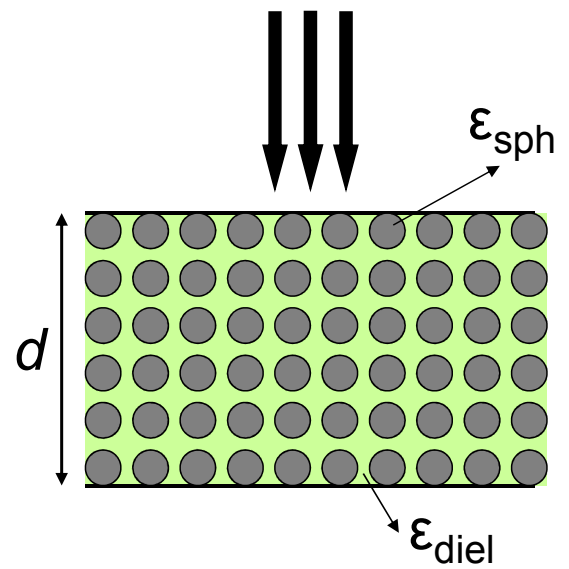

(a)

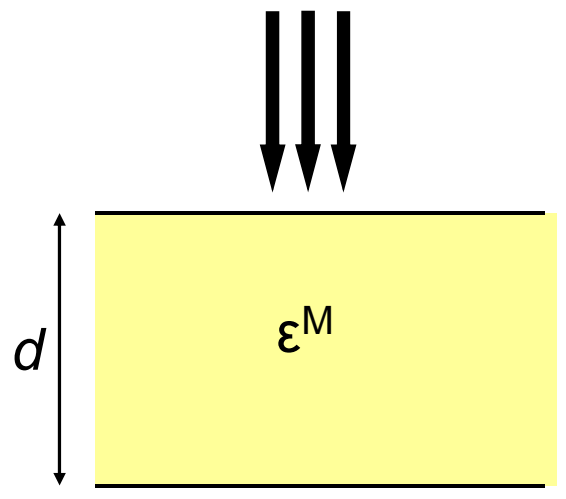

(b)

Figure 3. Scheme of the nanocomposite free standing film (thickness $d$ ). The black arrows indicate the direction of the incident field. (a) KKR: composite slab of metallic spheres of permittivity $\epsilon_{\mathrm{sph}}$ immersed in a dielectric host medium of permittivity $\epsilon_{\text {diel }}$. In the figure, the number of layers of spheres is $N=6$. (b) ENR: slab of a homogeneous material characterized by the macroscopic dielectric function $\epsilon^{M}$.

\section{Optical properties of nanocomposite thin films}

In this section we analyze the electromagnetic response of a slab made of a composite material. We first use the KKR to investigate the influence of the number of layers of spheres in its optical properties and establish the conditions for which the results given by the KKR permit a valid comparison with those of the effective medium theory (ENR). In Fig. 3 we show the scheme of the composite slab modeled with the KKR (a) and with the ENR (b).

\subsection{The KKR method}

As stated in the Introduction, among the methods available for the calculation of the electromagnetic response of composite periodic structures made of spheres, the KKR appears to be numerically efficient. Within its framework, the electromagnetic interactions between the inclusions arranged in the periodic lattice are calculated by means of the layer-multiple scattering method for spherical scatterers [38, 39, 40, 41]. The crystal can be considered as a stack of parallel layers formed by spheres periodically arranged in a 2D Bravais lattice. To solve the electromagnetic problem, the multiple scattering between spheres of each single layer is calculated first. Then, the scattered response of multiple layers is determined by using a procedure similar to the one used to calculate the reflection and transmission properties of stratified media with planar interfaces. The computer program MULTEM [39, 41] is a numerical implementation of the KKR. 
As mentioned in Section 2, to compute the optical response by the ENR is enough to set the filling fraction of the structure. On the other hand, to perform the simulations using MULTEM, the number of layers of spheres within the slab $(N)$ is also required. As a consequence, if we fix the slab thickness $(d)$ and the filling fraction of the nanomaterial, the lattice parameter and the radius of the spheres are determined by the relations $L=d / N$ and $r=L(3 f / 4 \pi)^{1 / 3}$ (for a simple cubic lattice of non-overlapping spheres), respectively. This is a very important aspect to remark, as it implies that for a given $d$, by varying $N$, it is possible to find different pairs of values $r, L$ that give the same $f$.

To investigate the influence of the number of layers for fixed values of $d$ and $f$, we plot the reflectance and the absorptance (Fig. 4) as a function of the photon energy $\hbar \omega$ for $d=360 \mathrm{~nm}$ and $1820 \mathrm{~nm}$. The different curves in each panel correspond to different values of $N$. For both thicknesses, we consider two different filling fractions, $f=0.045$ (top panel) and $f=0.23$ (bottom panel).

With increasing $N$ (for fixed values of $f$ and $d$ ), the radius of the spheres becomes smaller, the wavelength-to-radius ratio increases, and the long wavelength condition (in which the material behaves as a homogeneous medium) is achieved. Then, it is to expect that for increasing $N$ the curves tend to a limit response given by the long wavelength condition. This behavior can be observed in Fig. 4. In Table 1 we summarize the values of $L$ and $r$ for the slabs investigated in the present example. According to the values of $r$ given in Table 1, we can conclude that 32 layers for $d=360 \mathrm{~nm}$ and 128 layers for $d=1820 \mathrm{~nm}$ are sufficient to ensure the long wavelength condition within the spectral range considered.

Table 1. Geometrical parameters of the composite slab considered in Fig. 4 .

\begin{tabular}{|c|c|c|c|c|}
\hline$d(\mathrm{~nm})$ & $N$ & $L(\mathrm{~nm})$ & $r(\mathrm{~nm})(f=0.045)$ & $r(\mathrm{~nm})(f=0.23)$ \\
\hline \multirow{3}{*}{360} & 8 & 45 & 9.9 & 17.1 \\
& 16 & 22.5 & 4.95 & 8.55 \\
& 32 & 11.25 & 2.47 & 4.27 \\
\hline \multirow{3}{*}{1820} & 32 & 56.87 & 12.51 & 21.61 \\
& 64 & 28.44 & 6.25 & 10.80 \\
& 128 & 14.22 & 3.14 & 5.41 \\
\hline
\end{tabular}

\subsection{Comparison between the $K K R$ and the ENR}

We now consider a plane wave normally incident on a free standing film made of a composite medium of silver particles embedded in titanium dioxide, as that considered in Fig. 2(a). Figure 5 display the reflectance, absorptance and transmittance for two filling fractions, $f=0.045$ (top panel) and $f=0.23$ (bottom panel), and for $d=360$ $\mathrm{nm}$ (a) and $1820 \mathrm{~nm}$ (b). We present curves obtained using three different approaches: KKR, ENR and MG. For all the ENR calculations we used $M=120$. Taking into 

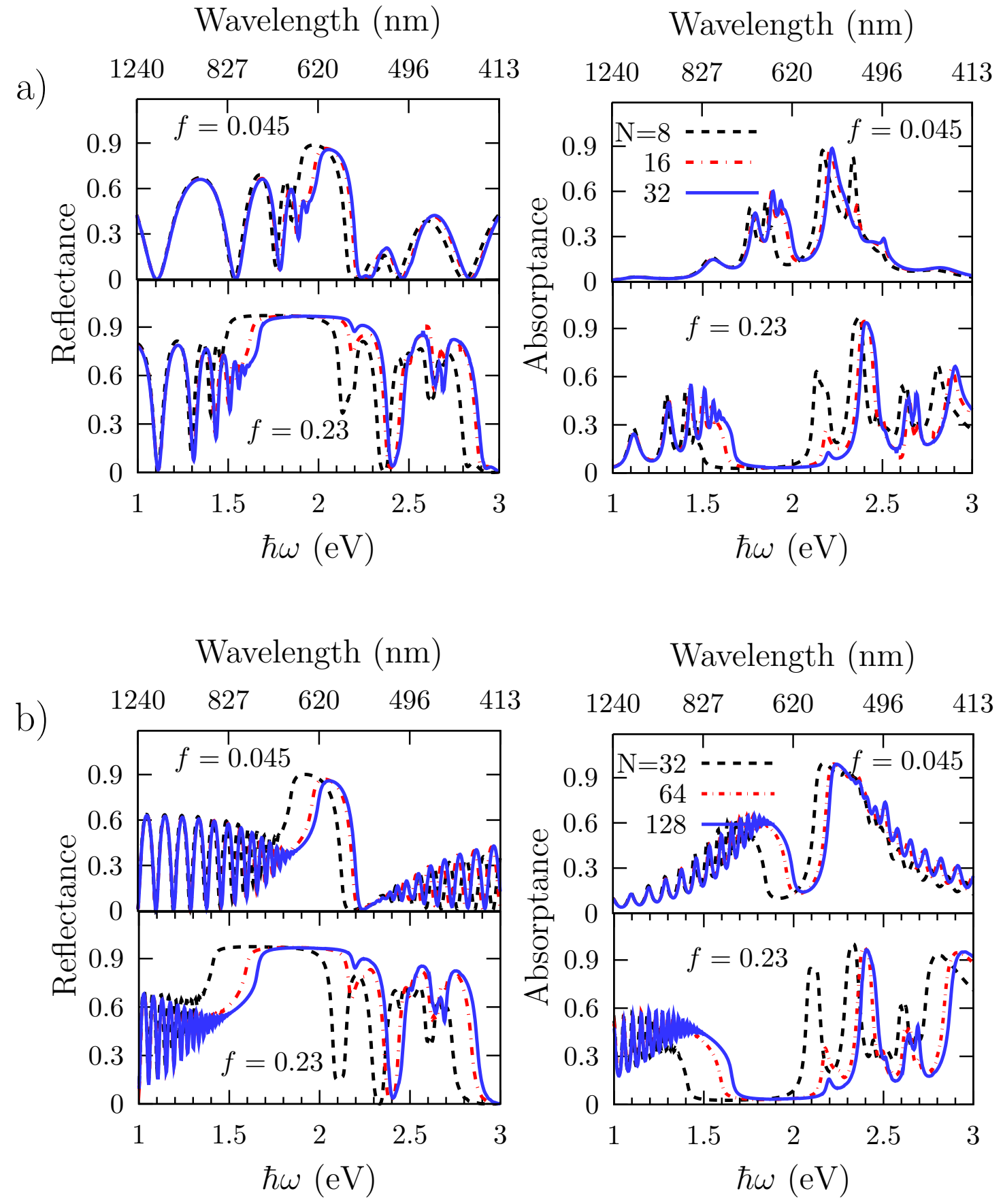

Figure 4. Reflectance (left panel) and absorptance (right panel) of a composite slab made of silver spheres embedded in a dielectric matrix of $\epsilon_{\text {diel }}=7.84$. a) $d=360 \mathrm{~nm}$ (dashed, dot-dashed and solid lines, correspond to $N=8,16$ and 32 , respectively); b) $d=1820 \mathrm{~nm}$ (dashed, dot-dashed and solid lines, correspond to $N=32,64$ and 128, respectively). Top (bottom) panels correspond to $f=0.045(f=0.23)$. 


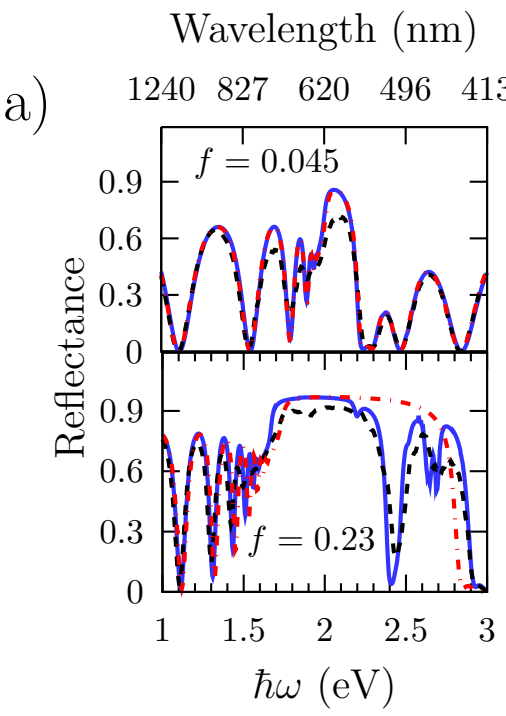

a)

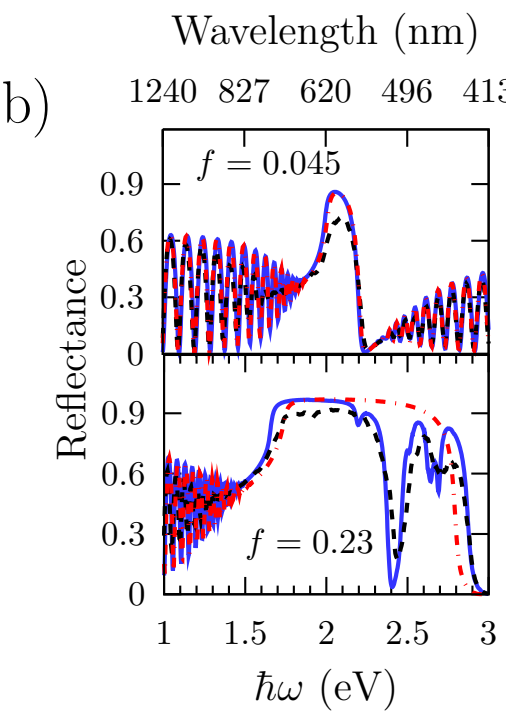

Wavelength (nm)

$\begin{array}{lllll}1240 & 827 & 620 & 496 & 413\end{array}$

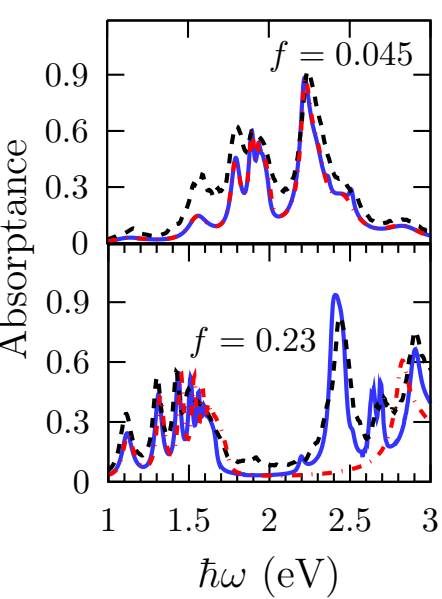

Wavelength (nm)

$\begin{array}{lllll}1240 & 827 & 620 & 496 & 413\end{array}$

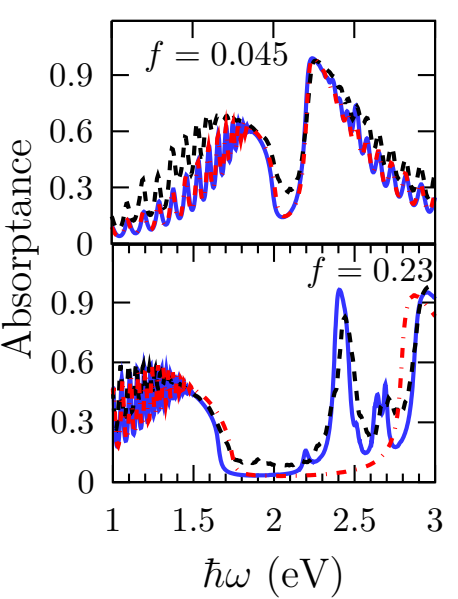

Wavelength (nm)

$\begin{array}{lllll}1240 & 827 & 620 & 496 & 413\end{array}$

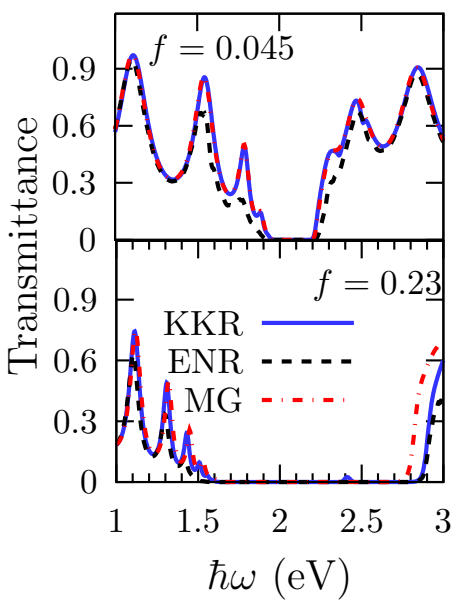

Wavelength (nm)

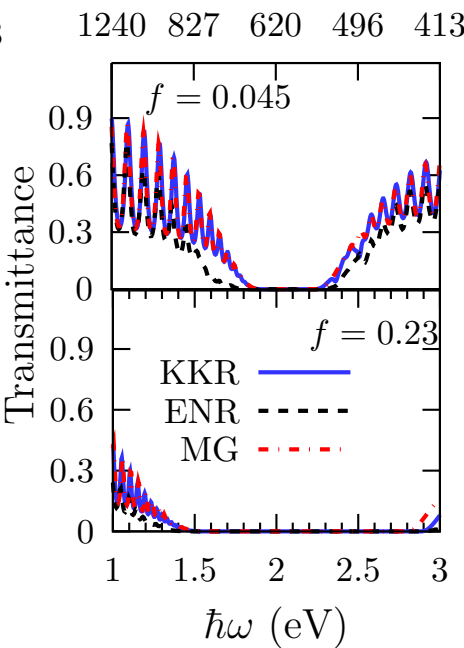

Figure 5. Reflectance (left panel), absorptance (center panel) and transmittance (right panels ) of a composite slab made of silver spheres embedded in a dielectric matrix of $\epsilon_{\text {diel }}=7.84$. a) $d=360 \mathrm{~nm}$ and b) $d=1820 \mathrm{~nm}$. Top (bottom) panels correspond to $f=0.045(f=0.23)$. Solid, dashed, and dot-dashed lines correspond to KKR, ENR, and MG, respectively.

account the analysis of Section 3.1, for the KKR simulations we used $N=32$ for $d$ $=360 \mathrm{~nm}$ and $N=128$ for $d=1820 \mathrm{~nm}$. The electromagnetic response of the slab is closely linked to the macroscopic dielectric function of the composite, i.e., to the behavior of $\operatorname{Re}\left\{\epsilon^{M}\right\}$ and $\operatorname{Im}\left\{\epsilon^{M}\right\}$, which are strongly frecuency dependent (see Fig. 2). According to this, the response of the slab exhibits very different features in the different spectral regions. At low frequencies, the curves for both values of $f$ present oscillations due to the Fabry-Perot interference and, as expected, for the thicker slab the adjacent maxima of these oscillations are closer to each other. In this spectral region $(\hbar \omega<2$ 
$\mathrm{eV}$ for $f=0.045$ and $\hbar \omega<1.7 \mathrm{eV}$ for $f=0.23$ ), $\operatorname{Re}\left\{\epsilon^{M}\right\}>0$ (as shown in Fig. 2 (a)) and therefore, the effective medium behaves as a lossy transparent material that allows transmission through the slab. However, when approaching the dipole resonance frequency from the low frequency side, $\operatorname{Re}\left\{\epsilon^{M}\right\}$ and $\operatorname{Im}\left\{\epsilon^{M}\right\}$ increase, and as a result, a lower transmittance is observed. This behavior becomes more evident for the thicker slab as observed in the right panels of Fig. 5 (b), in the ranges $1.8 \mathrm{eV}<\hbar \omega<2 \mathrm{eV}$ for $f=0.045$ and $1.5 \mathrm{eV}<\hbar \omega<1.7 \mathrm{eV}$ for $f=0.23$. It can be observed that for both thicknesses considered, there is a reflectance band that starts at the dipole resonance frequency: at $\approx 2 \mathrm{eV}(620 \mathrm{~nm})$ for $f=0.045$ and at $\approx 1.7 \mathrm{eV}(729.4 \mathrm{~nm})$ for $f=$ 0.23 (see left panels of Fig. 5). Within this band, the reflectance is very high (more than $80 \%$ ) and the transmittance is negligible. Notice that at the dipole resonance $\operatorname{Re}\left\{\epsilon^{M}\right\}$ changes its sign from positive to negative and stays negative in a frequency range that defines the reflectance bandwidth. This band is wider for $f=0.23$ (1.7 $\mathrm{eV}<\hbar \omega<2.9 \mathrm{eV})$ than for $f=0.045(2 \mathrm{eV}<\hbar \omega<2.2 \mathrm{eV})$ because the dipolar interaction is stronger for higher concentrations of spheres, as observed in Fig. 2 (a). Besides, for $f=0.23$ there is a pronounced dip within the reflectance band very close to a multipole resonance of higher order, which occurs at $\approx 2.5 \mathrm{eV}$. This dip is located within a narrow frequency range at which $\operatorname{Re}\left\{\epsilon^{M}\right\}$ is positive (see Fig. 2 (a)) and in this range the effective medium behaves as a lossy transparent material which allows light propagation. However, the electromagnetic response in this region differs substantially from that exhibited in the low frequency zone, in which the condition $\operatorname{Re}\left\{\epsilon^{M}\right\}>0$ also holds. In the vicinity of the multipole resonance, $\operatorname{Re}\left\{\epsilon^{M}\right\} \approx 1$ and therefore, the contrast between the macroscopic permittivity of the slab and that of vacuum is very low. Consequently, the reflectance becomes negligible and most of the incident energy enters the slab and is largely absorbed, although in this region $\operatorname{Im}\left\{\epsilon^{M}\right\}$ is not as high as in the dipole resonance. This effect has already been reported in 2D [21] and 3D structures [23].

In general, the qualitative agreement between the ENR and the KKR curves is satisfactory. Both methods adequately describe the spectral response of the composite slab. However, there are some quantitative differences in certain spectral ranges which could be due to the discretization of the sphere in the ENR. The surface roughness of the discretized particles produces modes that affect the electromagnetic response. For $f=0.045$, the KKR and the MG curves are in excellent agreement, as expected for a diluted system of spheres. However, for $f=0.23$, the MG does not predict the splitting of the reflectance band because it does not take into account multipolar interactions of higher orders. Conversely, since the KKR and the ENR consider in their formulations multipolar interactions between the spheres, they adequately describe the splitting of the reflection band at those frequencies at which multipole resonances are excited.

From the point of view of potential applications, compound metal-dielectric films can be used as tunable frequency light absorbers [47] by appropriately choosing $f$ and $\epsilon_{\text {diel }}$. 


\section{Application example: tuning of the absorption bands}

In this section we show that by properly combining the filling fraction of the nanoparticles array and the dielectric constant of the host medium, composite films can be used as tunable frequency light absorbers. Fig. 6 displays the absorptance vs. frequency of a composite slab made of silver spheres embedded in titanium dioxide (as in the previous examples) for different values of $f$, from $f=0.05$ (diluted composite of metallic spheres) up to $f=0.9$ (interpenetrated spheres). The slab is illuminated under normal incidence and its thickness is $1820 \mathrm{~nm}$.

For all the values of $f$, within the reflection band the absorptance is less than $10 \%$. When $f$ increases, the spectral position of this band shifts towards the red region of the spectrum and its bandwidth changes. Also, there is an absorption band at which the absorptance is $>90 \%$. This band is located at $\hbar \omega \approx 2.2 \mathrm{eV}(564 \mathrm{~nm})$ for $f \approx 0.05$ and its position shifts to the high frequency region as $f$ increases, being at $\hbar \omega \approx 3 \mathrm{eV}(412 \mathrm{~nm})$ for $f \approx 0.25$. From $f \approx 0.15$ up to $f=0.5$, there is another absorption peak at the frequency of splitting of the reflection band that appears as a consequence of the excitation of a multipole resonance of higher order [27]. The spectral position of this peak strongly depends on the value of $f$ and redshifts as $f$ increases. For $f=\pi / 6$ (close packing condition) the system percolates and becomes conducting at low frequencies. For larger $f$, the spheres interpenetrate and the interaction among the dielectric voids produces an absorption band $(\approx 90 \%)$ due to the index matching between the macroscopic dielectric function and air at NIR resonant frequencies. As $f$ increases, this band shifts to higher frequencies, as expected.

So far we have investigated the absorptance of the slab by considering different filling fractions for a fixed value of the dielectric constant of the host medium. To investigate the influence of $\epsilon_{\text {diel }}$ in the distribution of the absorption bands, we show in Fig. 7 the absorptance of a composite slab of silver nanoparticles as a function of $\epsilon_{\text {diel }}$ for $f=0.23$. As $\epsilon_{\text {diel }}$ increases, the multipole resonances, and therefore the absorption bands, shift to the red region of the spectrum, whereas its bandwidth remains approximately constant.

These examples evidence that the ENR constitutes a useful and powerful tool for the design of tunable absorbers based on metallic nanoparticle composites: by appropriately selecting the filling fraction and the permittivity of the host medium, the electromagnetic response can be tuned to obtain, for example, absorption peaks

and negligible transmittance and reflectance in the visible range of the electromagnetic spectrum.

\section{Conclusions}

We have considered a composite material comprising periodically distributed metallic spheres in a dielectric host matrix. The capabilities of the ENR approach to calculate its effective macroscopic dielectric function have been investigated. We have compared 


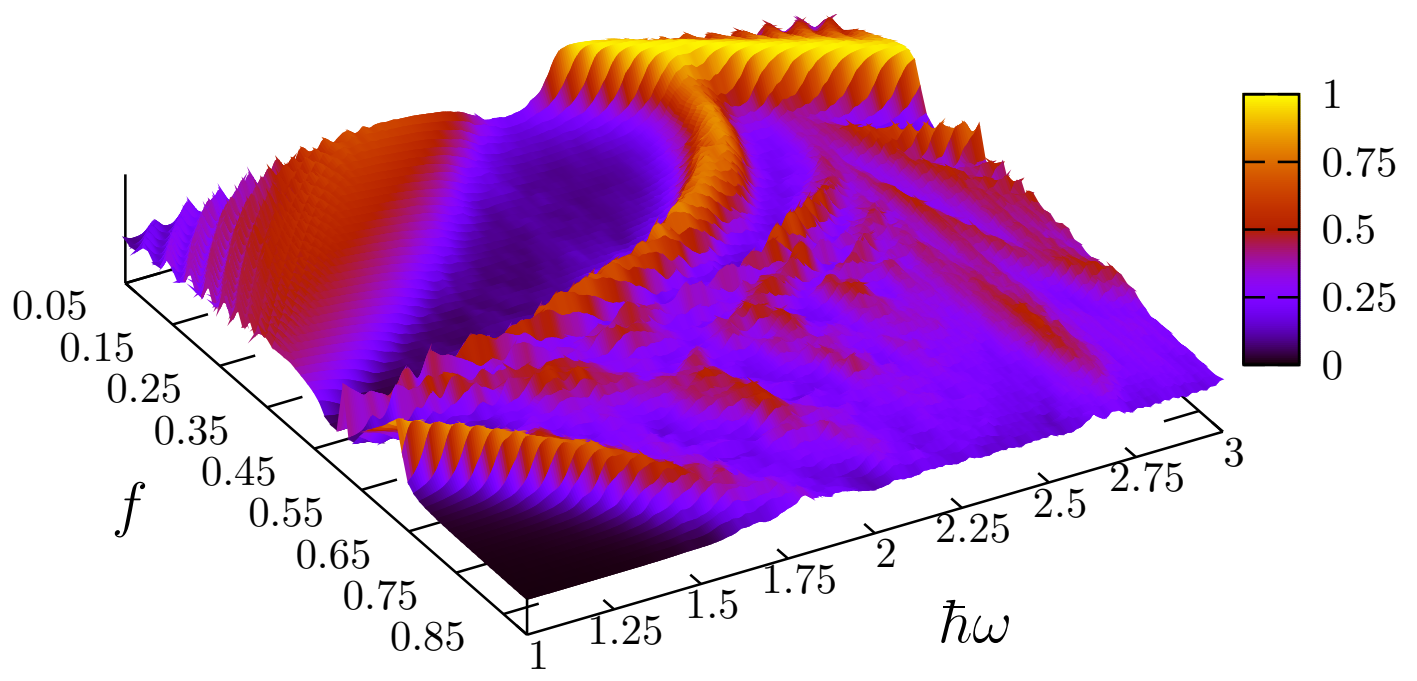

Figure 6. Absorptance of a composite slab made of silver spheres embedded in a dielectric matrix of $\epsilon_{\text {diel }}=7.84$ versus $f$ and $\hbar \omega$.

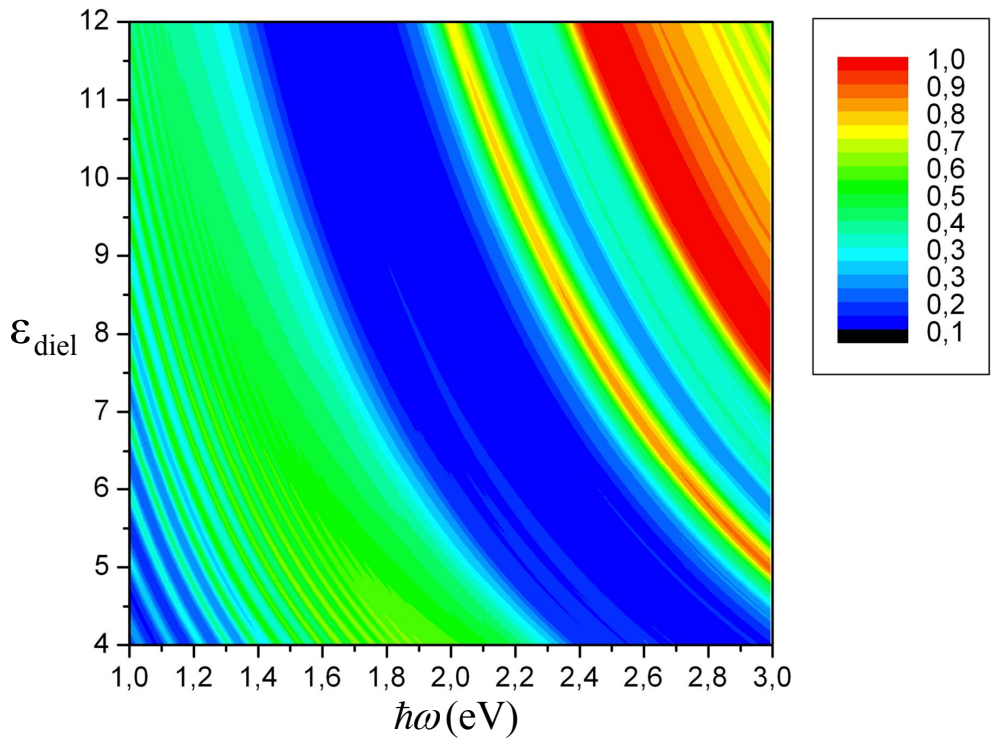

Figure 7. Absorptance of a composite slab made of silver spheres for $f=0.23$ versus $\epsilon_{\text {diel }}$ and $\hbar \omega$. 
the optical response of a composite slab obtained by the ENR with that of the KKR. Within the KKR framework, we have shown that for a fixed thickness, if the system comprises a sufficiently large number of layers, the radius of the spheres is small enough to guarantee the long wavelength approximation, and then a valid comparison between both methods can be done.

Both methods adequately describe the spectral response of composite slabs and the qualitative agreement between the curves is satisfactory. We analyzed in detail the behavior of the electromagnetic response of the slab in the different frequency regions and its relationship with the macroscopic dielectric function. We have found characteristic features in the response such as reflection and absorption bands whose spectral positions and widths can be controlled by the filling fraction and the permittivities of the particles and of the host medium. It is worth mentioning that although the faceted shape of the discretized particles is a drawback for modeling spherical inclusions, it can also be viewed as an advantage to study the optical features of synthesized metallic nanoparticles, which exhibit facets as a consequence of the manufacturing process [48, 49, 50]. Then, the ENR constitutes a versatile tool to investigate the optical properties of metallic nanoparticle composites.

\section{Acknowledgment}

This work was supported Agencia Nacional de Promoción Científica y Tecnológica (FONCYT-UNNE PICT-PRH-135-2008 (G.O.)), Consejo Nacional de Investigaciones Científicas y Técnicas (PIP 112-200801-01880 (D.S. and M.I.)), Universidad de Buenos Aires (UBA-20020100100533 (D.S. and M.I.) and (UBA-20020100100327 (R.D.)), and Universidad Nacional Autónoma de México (DGAPA-IN108413 (L.M.)).

\section{References}

[1] D. J. Bergman. Calculation of bounds for some average bulk properties of composite materials. Phys. Rev. B, 14(10):4304-4312, November 1976.

[2] R. Fuchs. Optical properties of small-particle composites. In J. C. Garland and D. B. Tanner, editors, Electrical Transport and Optical Properties of Inhomogeneous Media, number 40, AIP Conference Proceeding, pages 276-281. American Institute of Physics, 1977.

[3] G. W. Milton. Bounds on the complex dielectric constant of a composite material. Appl. Phys. Lett, 37:300-302, 1980.

[4] W. L. Mochán and R. G. Barrera. Electromagnetic response of systems with spatial fluctuations. i. general formalism. Phys. Rev. B, 32:4984-4988, 1985.

[5] W. L. Mochán and R. G. Barrera. Electromagnetic response of systems with spatial fluctuations. ii. applications. Phys. Rev. B, 32:4989-5001, 1985.

[6] R. G. Barrera, G. M. Monsivais, and W. L. Mochán. Renormalized polarizability in the maxwellgarnett theory. Phys. Rev. B, 38:5371-5379, 1988.

[7] K. Ghosh and R. Fuchs. Critical behavior in the dielectric properties of random self-similar composites. Phys. Rev. B, 44:7330-7343, 1991.

[8] B. U. Felderhof, G. W. Ford, and E. G. D. Cohen. Cluster expansion for the dielectric constant of a polarizable suspension. J. Stat. Phys., 28:135-164, 1982. 
[9] R. G. Barrera, G. M. Monsivais, W. L. Mochán, and E. Anda. Diagrammatic approach to the effective dielectric response of composite. Phys. Rev. B, 39(14):9998-10008, May 1989.

[10] B. T. Draine. Discrete-dipole aproximation for scattering calculations. J. Opt. Soc. Am. A, 11(4):1491-1499, April 1994.

[11] N. G. Khlebtsov, L. A. Dykman, Y. M. Krasnov, and A. G. Mel'nikov. Light absorption by the cluster of colloidal gold silver particles formed during slow and fast aggregation. Colloid Journal, $62: 765,2000$.

[12] K. K. Karkkainen, A. H. Sihvola, and K. I. Nikoskinen. Effective permittivity of mixtures: numerical validation by the FDTD method. Geoscience and Remote Sensing, IEEE Transactions on, 38:1303-1308, 2000.

[13] E. Lidorikis, S. Egusa, and J. D. Joannopoulos. Effective medium properties and photonic crystal superstructures of metallic nanoparticle arrays. J. Appl. Phys., 101:054304, 2007.

[14] M. Askari, N. Niakan, and A. Zakery. A high transmission and low loss metamaterial with negative refraction at 458 thz. Optik - International Journal for Light and Electron Optics, (0):-, 2012.

[15] O. Hess, J. B. Pendry, S. A. Maier, R. F. Oulton, J. M. Hamm, and K. L. Tsakmakidis. Active nanoplasmonic metamaterials. Nature Materials, 11:573, 2012.

[16] J. C. Garland and D. B. Tanner, editors. Electrical Transport and Optical Properties of Inhomogeneous Media, AIP Conference Proceeding No. 40. American Institute of Physics, New York, 1978.

[17] W. Luis Mochán and R. G. Barrera, editors. Electrical Transport and Optical Properties of Inhomogeneous Media, Physica A 207, Num 1-3. Elsevier, The Netherlands, 1994.

[18] G. W. Milton, K. M. Golden, D. Dobson, and A. Z. Vardeny, editors. Electrical Transport and Optical Properties of Inhomogeneous Media, Physica B 338, Num 1-4. Elsevier, North-Holland, 2003.

[19] M. G. Silveirinha. Metamaterial homogenization approach with application to the characterization of microstructured composites with negative parameters. Phys. Rev. B, 75:115104, 2007.

[20] J. S. Pérez-Huerta, Guillermo P. Ortiz, Bernardo S. Mendoza, and W. Luis Mochán. Macroscopic optical response and photonic bands. New J. Phys., 15(4):043037, 2013.

[21] Guillermo P. Ortiz, Brenda E. Martínez-Zérega, Bernardo S. Mendoza, and W.Luis Mochán. Effective dielectric response of metamaterials. Phys. Rev. B, 79:245132, 2009.

[22] E. Cortes, W. Luis Mochán, B. S. Mendoza, and G. P. Ortiz. Optical properties of nanostructured metamaterials. Phys. Status Solidi B, 247(8):2102-2107, 2010.

[23] W. Luis Mochán, Guillermo P. Ortiz, and Bernardo S. Mendoza. Efficient homogenization procedure for the calculation of optical properties of 3D nanostructured composites. Opt. Express, 18:22119-22127, 2010.

[24] D. A. G. Bruggeman. Berechnung verschiedener physikalischer konstanten von heterogenen substanzen. i. dielektrizitätskonstanten und leitfähigkeiten der mischkörper aus isotropen substanzen. Ann. Phys., 416:665-679, 1935.

[25] J. C. Maxwell-Garnett. Colours in metal glasses and metal films. Philos. Trans. R. Soc. London A, 203:385-420, 1904.

[26] W. T. Doyle. The permitivity of cubic arrays of spheres. In J. C. Garland and D. B. Tanner, editors, Electrical Transport and Optical Properties of Inhomogeneous Media, number 40, AIP Conference Proceeding, page 300. American Institute of Physics, 1977.

[27] F. Claro. Theory of resonant modes in particulate matter. Phys. Rev. B, 30:4989, 1984.

[28] P. C. Waterman and N. E. Pedersen. Electromagnetic scattering by periodic array of particles. J. Appl. Phys., 59:2609-2618, 1986.

[29] Ruey Lin Chern and Xing Xiang Liu. Effective parameters and quasi-static resonances for periodic array of dielectric spheres. J. Opt. Soc. Am. B, 27:488-496, 2010.

[30] Alexander Moroz. Localized resonances of composite particles. J. Phys. Chem. C., 113:2160421610, 2009.

[31] Vassilios Yannopapas and Alexander Moroz. Negative refractive index metamaterials from 
inherently non-magnetic materials for deep infrared to terahertz frequency ranges. J. Phys.: Condens. Matter, 17:3717-3734, 2005.

[32] R. G. Barrera and C. I. Mendoza. Three-particle correlations in the optical properties of granular composites. Solar Energy Materials and Solar Cells, 32:463, 1994.

[33] Guillermo P. Ortiz, C. López-Bastidas, J. A. Maytorena, and W. Luis Mochán. Bulk response of composite from finite samples. Physica B, 338:54-57, 2003.

[34] R. Rojas and F. Claro. Electromagnetic response of an array of particles: normal-mode theory. Phys. Rev. B, 34:3730, 1986.

[35] Brian Geist, William B. Spillman Jr., and Richard O. Claus. Thermal cycling and the optical and electrical characterization of self-assembled multilayer nile blue a-gold thin films. Appl. Opt., 44(30):6357-6360, Oct 2005.

[36] Jin Hyoung Lee, Qi Wu, and Wounjhang Park. Metal nanocluster metamaterial fabricated by the colloidal self-assembly. Opt. Lett., 34(4):443-445, Feb 2009.

[37] S. Mühlig, C. Rockstuhl, V. Yannopapas, T. Bürgi, N. Shalkevich, and F. Lederer. Optical properties of a fabricated self-assembled bottom-up bulk metamaterial. Opt. Express, 19(10):9607-9616, May 2011.

[38] A. Modinos. Scattering of electromagnetic waves by a plane of spheres-formalism. Physica A, 141:575-588, 1987.

[39] N. Stefanou, V. Yannopapas, and A. Modinos. Heterostructures of photonic crystals: frequency bands and transmission coefficients. Comput. Phys. Commun., 113:49-77, 1998.

[40] V. Yannopapas, A. Modinos, and N. Stefanou. Optical properties of metallodielectric photonic crystals. Phys. Rev. B, 60:5359, 1999.

[41] N. Stefanou, V. Yannopapas, and A. Modinos. Multem 2: A new version of the program for transmission and band-structure calculations of photonic crystals. Comput. Phys. Commun., 132:189-196, 2000.

[42] L. A. Dorado, R. A. Depine, and H. Míguez. Effect of extinction on the high-energy optical response of photonic crystals. Phys. Rev. B, 75:241101, 2007.

[43] R. Haydock. The recursive solution of the Schrödinger equation. Solid State Physics, 35:215, 1980.

[44] P. B. Johnson and R. M. Christy. Optical constant of noble metals. Phys. Rev. B, 6:4370, 1972.

[45] K. Glazebrook, J. Brinchmann, J. Cerney, C. DeForest, D. Hunt, T. Jenness, T. Luka, R. Schwebel, and C. Soeller. Pdl: The perl data language v.2.4.4. Dr. Dobb's Journal, http://pdl.perl.org, 1997.

[46] U. Kreibig and M. Vollmer. Optical Properties of Metal Cluster. Springer, 1995.

[47] Petru Ghenuche, Grégory Vincent, Marine Laroche, Nathalie Bardou, Riad Haïdar, Jean-Luc Pelouard, and Stéphane Collin. Optical extinction in a single layer of nanorods. Phys. Rev. Lett., 109:143903, 2012.

[48] Z. L. Wang. Transmission electron microscopy of shape-controlled nanocrystals and their assemblies. J. Phys. Chem. B., 104:1153, 2000.

[49] M. J. Yacaman, J. A. Ascencio, H. B. Liu, and J. Gardea-Torresdey. Structure shape and stability of nanometric sized particles. J. Vac. Sci. Technol. B., 19:1091, 2001.

[50] A. L. Gonzalez, C. Noguez, G. P. Ortiz, and G. Rodriguez-Gattorno. Optical absorbance of colloidal suspensions of silver polyhedral nanoparticles. J. Phys. Chem. B, 109:17512-17517, 2005 . 